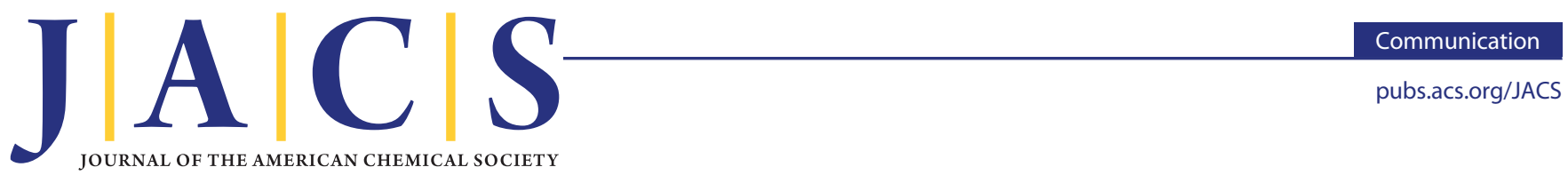

\title{
, Solvent-Free Strategy Yields Size and Shape-Uniform Capsules
}

\author{
2 Ana M. S. Costa and João F. Mano*๑ \\ 3 Department of Chemistry, CICECO, University of Aveiro, Campus Universitário de Santiago, 3810-193 Aveiro, Portugal \\ 4 S Supporting Information
}

\begin{abstract}
Capsules with a liquefied core were fabricated via the assembly of polymeric droplets induced by superamphiphobic surfaces. These highly repellent substrates exhibit distinct features such as (i) an easy and precise control over the particle size and shape, (ii) a high encapsulation efficiency, (iii) mild processing conditions, and (iv) the possibility to include any object in either a water or oil-based liquid core, which are not found on the current available strategies. As proof of concept, a photocross-linkable derivative of chitosan was used to produce the polymeric shell while a wealth variety of template cores were tested using a reversible cross-linking mechanism, interfacial gelation process or ice. Owing to the widespread application of polymeric capsules, the developed strategy is poised to usher the development of the next generation of materials not only for biomedical purposes but also for cosmetics, agriculture and electronics.
\end{abstract}

${ }_{21}^{21} \mathrm{~A}$ significant research interest is being devoted toward the use of hollow materials as encapsulation devices for a 24 plethora of different fields, spanning from electronics to 25 cosmetics, including biomedical applications. ${ }^{1}$ Core-shell 26 structured particles with a liquid core exhibit (i) a more 27 efficient and homogeneous transfer of solutes, (ii) a higher 28 loading capacity provided by their internal ample space, and 29 (iii) a lighter weight when comparing with their cross-linked30 core counterparts. ${ }^{2}$ Drawn by these appealing features, distinct 31 strategies to fabricate polymeric capsules have been devised. ${ }^{3}$ 32 However, most of them are based on complex and harsh 33 synthesis procedures, eluding the use of coagulating baths, 34 which can ultimately compromise the cargo stability and 35 loading efficiency. Thus, the absence of a simple and solvent36 free methodology to prepare liquid-core capsules under mild 37 conditions was the motivation of this work.

38 Herein, highly repellent substrates were used to design 39 monosized and spherical polymeric capsules with a (i) hydrogel 40 shell made of methacrylamide chitosan (MACHI), a biocom41 patible and light-sensitive derivative of $\mathrm{CHI}$, and (ii) a liquefied 42 core, wherein different molecules can be dispersed. Recently, 43 surfaces with low wettability were successfully employed to 44 produce compact spherical particles, from a wide range of 45 materials and under mild conditions, by cross-linking pregel 46 spherical droplets formed when in contact with these substrates 47 (SI; S1). ${ }^{4}$ However, the use of this solvent-free technology to 48 attain liquefied capsules have not been reported. ${ }^{5}$ First, the 49 liquefied core was obtained by dispensing a predefined volume 50 of an alginate (ALG) solution onto a SA surface, which is and oil-based liquids (Figure 1I and SI; S1.1). ALG was $52 \mathrm{fl}$ selected due to its biocompatibility as well as for its ability to 53

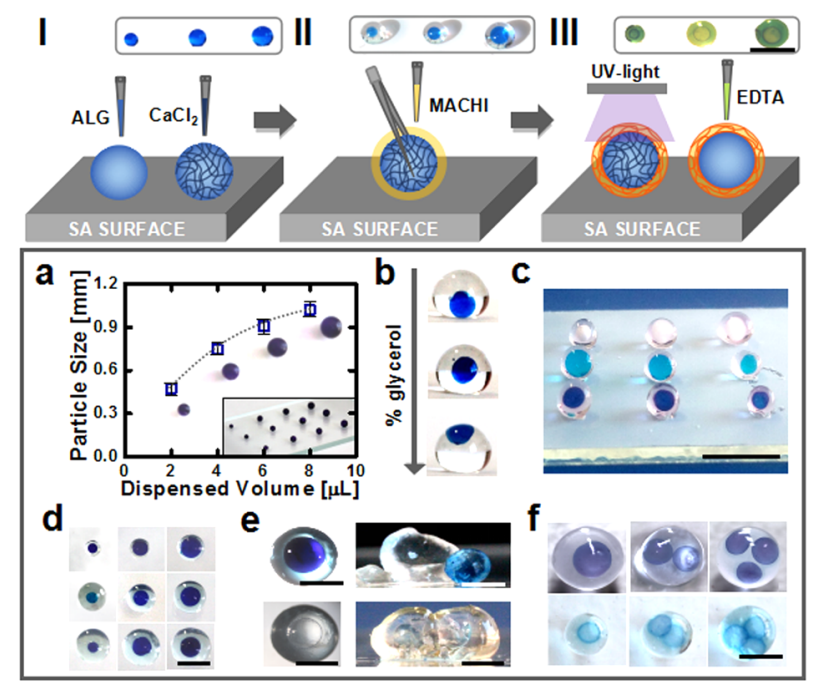

Figure 1. (I) Spherical ALG droplet induced by a SA surface and their subsequent $\mathrm{Ca}^{2+}$-mediated cross-linking. (II) Entrapment of a ALG core within a MACHI droplet. (III) UV-mediated cross-linking of the MACHI shell followed by the core dissolution via EDTA action. Scale bar stands for $1.2 \mathrm{~mm}$. (a) Effect of the dispensed volume of ALG solution on the size of the obtained ALG particles after $15 \mathrm{~min}$ of $\mathrm{Ca}^{2+}$-mediated gelling. Scale bar corresponds to $6 \mathrm{~mm}$. (b) Effect of glycerol on the position of the ALG core inside a MACHI pregel droplet (0, 16 and $20 \%(\mathrm{v} / \mathrm{v})$ of glycerol/water). (c) Scale-up of the developed strategy to attain simultaneously polymeric capsules containing cores with different sizes and entrapping different compounds. Scale bar stands for $2 \mathrm{~mm}$. (d) Multicompartmental hydrogel particles with distinct shell thickness. Scale bar is $1.2 \mathrm{~mm}$. (e) MACHI capsule before (upper panel) and after (lower panel) the EDTA treatment. Scale bar stands for $400 \mu \mathrm{m}$. (f) Hydrogel particles with a multicore structure before (upper panel) and after (lower panel) EDTA treatment. Scale bar corresponds to $700 \mu \mathrm{m}$.

reversibly form hydrogels at mild conditions, making it a great 54 candidate for the capsule liquid core (SI, S1.2). As shown in 55 Figure 1a, pregel ALG droplets remained suspended above SA 56 substrates, acquiring an almost spherical shape (SI; S1.1, shape 57 factor of $0.95 \pm 0.02$ ). This shape was induced by the extreme 58 wettability of these surfaces, which, in turn, is the result of the 59

Received: November 18, 2016

Published: January 10, 2017 
60 presence of a hierarchical topography with micro- and 61 nanofeatures and a low surface energy (SI; S1.3).

62 Afterward, calcium chloride $\left(\mathrm{CaCl}_{2}\right)$ was added above the 63 preformed ALG droplets to prompt their gelation (Figure 1I). 64 Figure 1a shows the possibility of controlling the ALG particle 65 size with high precision by simply tuning the dispensed volume 66 above the SA surface. By changing the droplet volume from 2 67 to $8 \mu \mathrm{L}$, the particle size increased from $0.5 \pm 0.05 \mathrm{~mm}$ to $1.0 \pm$ 680.05 after $15 \mathrm{~min}$ of $\mathrm{Ca}^{2+}$-mediated cross-linking, which 69 corroborates with previous studies $\left(R^{2} \approx 0.99\right)\left(\right.$ SI; S1.4). ${ }^{6}$

70 The preformed ALG hydrogel particles were then entrapped 71 within a larger volume of MACHI solution, previously 72 dispensed above a SA surface, to form a shell around it (Figure 73 1II). As shown in Figure 1b (upper panel), the ALG core sank 74 almost instantaneously onto the SA due to a density mismatch 75 between the MACHI solution (liquid) and the ALG particle 76 (hydrogel). Consequently, the external MACHI droplet may 77 not surround the core in the contact area with SA surface, 78 forming a hole from which the immobilized cargo may be 79 released in an uncontrolled way (SI; S1.4). To overcome this 80 main issue, glycerol was used to increase the density of the 81 external MACHI solution and compensate for the higher 82 density of the ALG particle. As can be observed in Figure 1b, 83 the ALG particle position inside the MACHI droplet can be 84 tuned by adjusting the amount of glycerol added. Moreover, the 85 inclusion of this compound ensures the scale-up of this process 86 to attain simultaneously polymeric capsules with different core 87 sizes (Figure 1c).

88 Afterward, the MACHI hydrogel shell was cross-linked upon 89 exposing this photosensitive polymer to UV-light for $1 \mathrm{~min}$ 90 (Figure 1III and SI; S1.5). By varying the dispensed volume of 91 MACHI polymer, the capsule thickness ranged from around 92100 to $400 \mu \mathrm{m}$ (Figure 1d).

93 Finally, the ALG core was dissolved upon dropping an 94 ethylenediaminetetraacetic acid (EDTA) solution above the 95 previous particle, yielding a MACHI capsule with a liquid core 96 (Figure 1III). EDTA, a divalent ion chelating agent, can disrupt 97 the $\mathrm{ALG} / \mathrm{Ca}^{2+}$ matrix as demonstrated by the conversion of the 98 ALG solid core into a liquid (Figure 1e and SI; S1.2). Further 99 control over the internal structure was demonstrated by 100 synthesizing capsules exhibiting multiple-cores (Figure 1f). 101 To produce these particles, different number of the preformed 102 ALG templates were assembled simultaneously within a droplet 103 of a MACHI precursor solution, which was subsequently gelled 104 by UV-light exposure (Figure 1f; upper panel) and its cores 105 liquefied upon EDTA action (Figure 1f; lower panel). Capsules 106 with a hierarchical architecture of more than two core 107 assemblies could be useful for individual reagent loading in 108 each of the created subcompartments, being attractive as 109 artificial organelles, bioreactors for confined synthesis or as 110 drug carries.

111 Capsules with a core-shell structure were subsequently 112 loaded with cells to assess the suitability of the purposed 113 strategy to encapsulate highly sensitive compounds. To this 114 end, the viability of human fibroblasts entrapped in five distinct 115 cell carrier formulations was assessed. First, cells were 116 homogeneously distributed within an $\mathrm{ALG} / \mathrm{Ca}^{2+}$ matrix, 117 exhibiting good viability rates due to (i) the mild processing 118 conditions used, (ii) the efficient exchange of essential 119 molecules with the surroundings provided by the particle 120 small size, and (iii) the ALG biocompatible character (Figure $1212 \mathrm{~A})$. Then, these cell carriers were entrapped within a second 122 polymeric layer made of MACHI (SI; S2). As shown on Figure
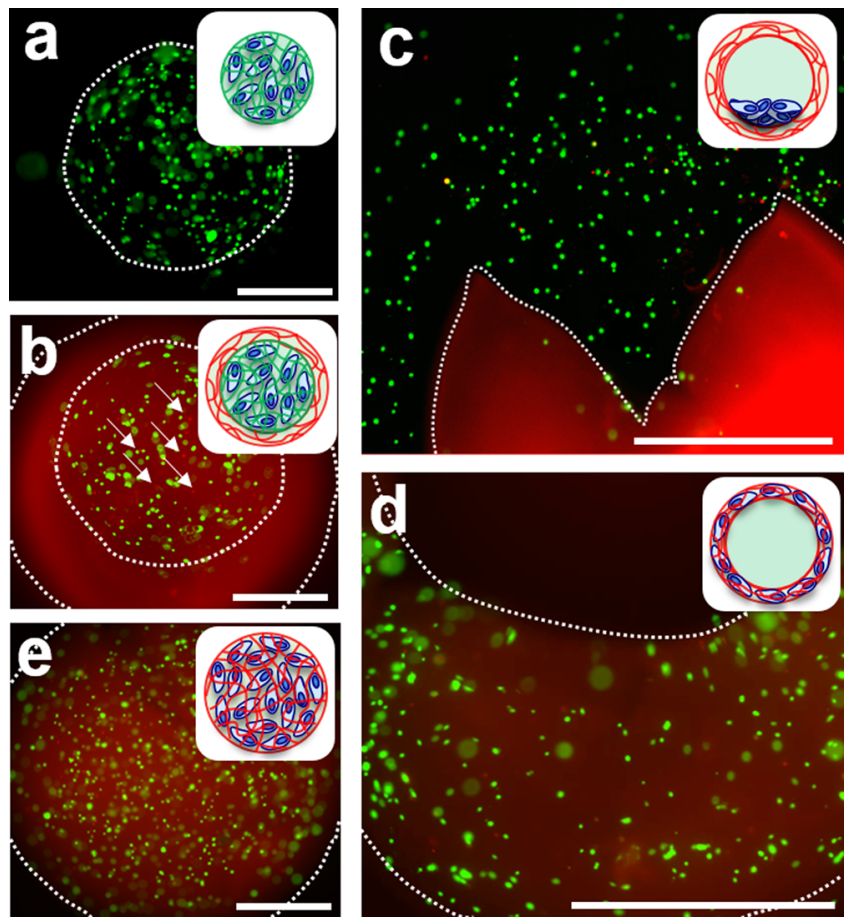

Figure 2. LIVE/DEAD images of cell-laden ALG microparticles (A), MACHI capsule with a cross-linked cell-laden ALG core (arrow indicates some nonviable cells) (B), a ruptured $\mathrm{MACHI}$ capsule releasing the encapsulated cells (C), cell-laden MACHI shell (D), and cell-laden compact MACHI particle (E) using calcein (green; living cells) and Ethd-1 (red; dead cells) dyes. Scale bar corresponds to 200 $\mu \mathrm{m}$.

$2 \mathrm{~B}$, some nonviable cells appeared on the core center after the 123 incorporation of this barrier between the core and the culture 124 medium. This may be ascribed to the increase of the overall 125 diameter of the particle, which hampered the diffusion of 126 nutrients $/ \mathrm{O}_{2} /$ cell residuals and, hence, compromised the cell 127 viability. Therefore, these particles were subjected to a EDTA 128 step to create liquefied cell-laden capsules. The results suggest 129 the formation of a cell-friendly liquid environment wherein cells 130 are metabolically active, highlighting the potential of these 131 capsules as cell encapsulation devices (Figure 2C). Indeed, 132 previous works have shown higher cell viability rates for higher 133 core dissolution degrees, which can justify the attained high 134 viability levels. ${ }^{8}$ Other alternative to enhance the diffusion rates 135 was tested by entrapping fibroblasts within the thin $(\approx 200 \mu \mathrm{m}) 136$ MACHI shell (Figure 2D). When comparing with compact 137 cell-laden MACHI particles, which revealed nonviable cells at 138 the inner areas (Figure 2E), most of the cells enclosed on the 139 MACHI shell were viable, which further strengths the potential 140 of the developed liquefied capsules.

Figure 3 summarizes different strategies to produce $142 \mathrm{f} 3$ polymeric capsules using different sacrificial cores along with 143 distinct removal methods. Gelatin, the denaturated form of 144 collagen protein, was used as a core due to its temperature- 145 responsive behavior. At low temperatures, its chains undergo a 146 conformational change from a random coil to a triple helix, 147 resulting on the formation of a 3D cross-linked network (Figure 148 3a). Interestingly, this aggregation process can be reversibly 149 disrupted above $30{ }^{\circ} \mathrm{C}$ to yield liquefied capsules (Figure $3 \mathrm{~b}$ ). ${ }^{9} \quad 150$ The use of gelatin as template constitutes a simplification over 151 the process described on Figure 1 because it avoids the addition 152 of any compound to either cross-link or liquefy the core. 153 

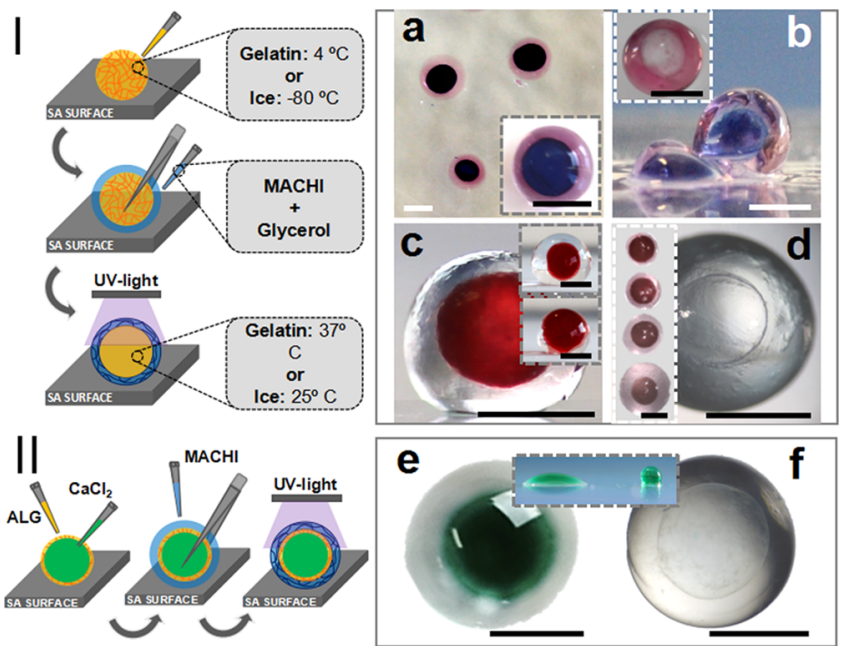

Figure 3. Fabrication of polymeric capsules: (I) Thermoresponsive sacrificial cores: examples of MACHI capsules with a gelatin $(a, b)$ or ice core $(\mathrm{c}, \mathrm{d})$ before $(\mathrm{a}, \mathrm{c})$ and after $(\mathrm{b}, \mathrm{d})$ the core removal, respectively. Regarding the ice-core capsules, ethanol was added to decrease the density of the surrounding MACHI solution (c, upper inset), the temperature was controlled to avoid the core melting (c, lower inset) and the shell thickness tuned by controlling the volume of MACHI solution dispensed (e, inset). (II) Interfacial gelation process: example of a MACHI capsule with a $\mathrm{CaCl}_{2}$ liquid-core before (e) and after (f) the dye release.

154 Another strategy also based on the use of thermosensitive 155 templates consists in using ice as template. Contrarily to cross156 linked cores, an ice core floats when placed above a MACHI 157 droplet (Figure 3c). Thus, ethanol was added to lower the 158 density of the surrounding droplet (Figure 3d). Such capsules 159 may be extremely attractive for the cryopreservation of living 160 cells, an issue that has received increasing attention. Contrarily 161 to some living organisms, most mammalian cells are unable to 162 survive when exposed to subzero temperatures unless they are 163 placed in solutions with specific additives and following defined 164 freezing protocols. ${ }^{10}$ Recently, the entrapment of the desired 165 structures inside hydrogels emerged as an alternative to the 166 established protocols since they allow the cell protection from 167 mechanical damage upon ice crystallization and preserve the 168 cell-cell interactions. ${ }^{11}$ With this in mind, polymeric capsules 169 containing both cells and cryopreservatives could be fabricated 170 following this methodology, envisioning cell preservation for 171 future outcomes. Polymeric capsules were also templated on a 172 liquid core by depositing a $\mathrm{CaCl}_{2}$ droplet above another of 173 ALG, resulting on a thin, elastic interfacial membrane (Figure 174 3II). Following this methodology, bicompartmental hydrogel 175 particles were formed by assembling this core inside a MA-CHI 176 shell (Figure 3e,f). Using this strategy, the addition of any 177 compound to adjust the density or to remove the core is 178 avoided.

179 Hierarchical systems were fabricated by incorporating 180 different objects inside the core during the synthesis process, 181 proving once more the versatility of this strategy. Herein, 182 calcium carbonate $\left(\mathrm{CaCO}_{3}\right)$ particles were evenly distributed 183 within the ALG core as visualized by an arrangement of white 184 dots, characteristic of these particles (Figure 4a). These 185 subcompartments can also be disrupted through the action of 186 EDTA, which turn the core into a liquid (Figure 4b). Such 187 compartmentalized systems may find biomedical utility, which 188 is imparted by their proven biocompatibility. ${ }^{12}$ Similarly, PLLA
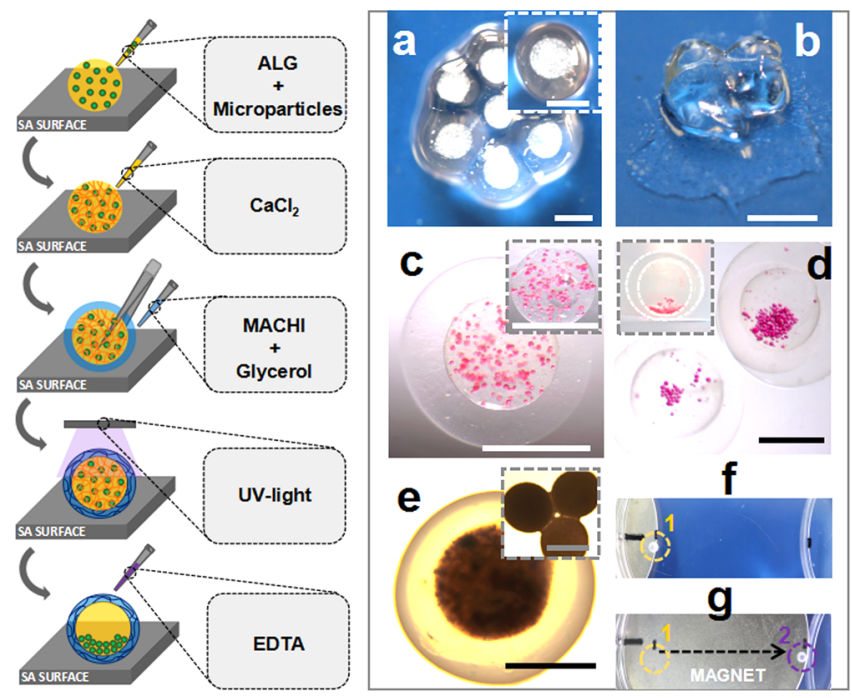

Figure 4. Fabrication of hierarchical capsules containing either $\mathrm{CaCO}_{3}$ $(\mathrm{a}, \mathrm{b})$ or PLLA microparticles $(\mathrm{c}, \mathrm{d})$, or $\mathrm{Fe}_{3} \mathrm{O}_{4}$ nanoparticles before $(\mathrm{a}, \mathrm{c}, \mathrm{e})$ and after (b,d,f,g) EDTA treatment. Motion control of the produced capsules using a permanent magnet (surface magnetic intensity of $0.075 \mathrm{~T} ; \mathrm{f}, \mathrm{g})$. Scale corresponds to $400 \mu \mathrm{m}$.

microparticles were also enclosed within MACHI capsules and 189 may have an increased importance, for example, as supporting 190 points of anchorage-dependent cells as they are enable to grow 191 in suspension (Figure 4c,d). Actually, it was previously reported 192 higher cell viability levels, around 50\%, when cells were 193 encapsulated within particles containing anchorage points, 194 highlighting the importance of these particulate devices for 195 application in Tissue Engineering rather than be merely used as 196 cell carriers. ${ }^{13}$ Furthermore, the stability of the obtained 197 capsules was assessed through a rotational test using capsules 198 with two layer thicknesses, i.e., 250 and $350 \mu \mathrm{m}$. Interestingly, 199 after $1 \mathrm{~h}$ at $200 \mathrm{rpm}$, both capsules maintained their integrity 200 avoiding the release of their contents, thus proving the 201 production of stable capsules. Furthermore, $\mathrm{Fe}_{4} \mathrm{O}_{3}$ particles 202 were successfully encapsulated within MACHI capsules (Figure 203 $4 \mathrm{e}$ ), empowering these capsules with magnetic-responsiveness 204 that can be used to guide them over a surface (Figure $4 \mathrm{f}$ and 205 $4 g)$.

In summary, SA surfaces were successfully employed to 207 fabricate ready-to-use and stable multiscaled liquefied capsules 208 enclosing different objects. This strategy benefit from its (i) 209 solvent-free character enabling a loading efficiency of almost 210 $100 \%$, (ii) reproducibility as demonstrated by the great control 211 over the particle size and shape, (iii) versatility as shown by the 212 fabrication of a wide variety of core-shell capsules, (iv) mild 213 processing conditions as proved by the safe encapsulation of 214 metabolically active cells, and (v) its cost-effective character 215 inasmuch as it is based on a simple setup. Based on all these 216 features, this simple, yet efficient strategy is envisioned to 217 constitute an innovative approach to produce liquid-core 218 polymeric systems to entrap a variety of sensitive molecules 219 including not only cells but also proteins, genes, enzymes, and 220 drugs, with minimal adverse effects on their functionality. 221 Moreover, due to the simultaneously superhydrophobic and 222 superoleophobic character of the used substrates, capsules may 223 contain virtually any type of liquid make it possible to broad the 224 application spectrum to diverse technological purposes such as 225 agriculture, biotechnology, cosmetics, and electronics, where 226 
227 solvents different than water are often required. Owing to the 228 widespread application of polymeric capsules like the produced 229 ones, modifications to the conventional fabrication techniques 230 are likely to have a strong impact and open new prospects for 231 the development of the next generation of engineered 232 polymeric assemblies for both science and technology.

\section{$233 \square$ ASSOCIATED CONTENT}

234 S Supporting Information

235 The Supporting Information is available free of charge on the 236 ACS Publications website at DOI: 10.1021/jacs.6b11925.

237 Preparation and characterization of the polymeric 238 capsules, the superamphiphobic surfaces, the methacry239 lamide chitosan, cell carriers, and hierarchical polymeric 240 capsules (PDF)

\section{AUTHOR INFORMATION}

\section{Corresponding Author}

243 *J. F. Mano. E-mail: jmano@ua.pt

244 ORCID

245 João F. Mano: 0000-0002-2342-3765

246 Notes

247 The authors declare no competing financial interest.

\section{$248 \square$ ACKNOWLEDGMENTS}

249 A.M.S.C. thanks Fundação para a Ciência e Tecnologia (FCT) 250 for the financial support through the Ph.D. grant (SFRH/BD/ 251 101748/2014).

\section{$252 \square$ REFERENCES}

253 (1) (a) Lou, X. W.; Archer, L. A.; Yang, Z. Adv. Mater. 2008, 20, 254 3987. (b) Tibbitt, M. W.; Dahlman, J. E.; Langer, R. J. Am. Chem. Soc. 255 2008, 130, 15808. (c) Blaiszik, B. J.; Kramer, S. L. B.; Grady, M. E.; 256 McIlroy, D. A.; Moore, J. S.; Sottos, N. R.; White, S. R. Adv. Mater. 257 2012, 24, 398. (d) Lam, P. L.; Gambari, R. J. Controlled Release 2014, $258178,25$.

259 (2) (a) Lima, A. C.; Alvarez-Lorenzo, C.; Mano, J. F. Adv. Healthcare 260 Mater. 2016, 5, 1687. (b) Verma, S. K.; Amoah, A.; Schellhaas, U.; 261 Winterhalter, M.; Springer, S.; Kolesnikova, T. A. Adv. Funct. Mater. 262 2016, 26, 6015.

263 (3) Cui, J.; van Koeverden, M. P.; Müllner, M.; Kempe, K.; Caruso, 264 F. Adv. Colloid Interface Sci. 2014, 207, 14.

265 (4) (a) Song, W.; Lima, A. C.; Mano, J. F. Soft Matter 2010, 6, 5868. 266 (b) Costa, A. M. S.; Alatorre-Meda, M.; Oliveira, N. M.; Mano, J. F. 267 Langmuir 2014, 30, 4535.

268 (5) Lima, A. C.; Mano, J. F. Nanomedicine 2015, 10, 271.

269 (6) Luo, R.; Cao, Y.; Shi, P.; Chen, C. H. Small 2014, 10, 4886.

270 (7) (a) Peters, R. J. R. W.; Louzao, I.; van Hest, J. C. M. Chem. Sci.

271 2012, 3, 335. (b) Xu, W.; Ledin, P. A.; Iatridi, Z.; Tsitsilianis, C.; 272 Tsukruk, V. V. Angew. Chem., Int. Ed. 2016, 55, 4908. (c) Costa, A. M. 273 S.; Alatorre-Meda, M.; Alvarez-Lorenzo, C.; Mano, J. F. Small 2015, $27411,3648$.

275 (8) Broughton, R. L.; Sefton, M. V. Biomaterials 1989, 10, 462.

276 (9) Duconseille, A.; Astruc, T.; Quintana, N.; Meersman, F.; Sante277 Lhoutellier, V. Food Hydrocolloids 2015, 43, 360.

278 (10) Shaw, J. M.; Oranratnachai, A.; Trounson, A. O. Theriogenology 279 2000, 53, 59.

280 (11) Nie, Y.; Bergendahl, V.; Hei, D. J.; Jones, J. M.; Palecek, S. P. 281 Biotechnol. Prog. 2009, 25, 20.

282 (12) Wei, W.; Ma, G.-H.; Hu, G.; Yu, D.; McLeish, T.; Su, Z.-G.; 283 Shen, Z.-Y. J. Am. Chem. Soc. 2008, 130, 15808.

284 (13) Correia, C. R.; Sher, P.; Reis, R. L.; Mano, J. F. Soft Matter 2013, $2859,2125$. 